\title{
Nonmetallic plasmonic heterostructures with multi- synergies on boosting hot electron generation for CO2 photoreduction
}

\section{Zaizhu Lou ( $\square$ zzlou@jnu.edu.cn ) \\ Jinan University}

Changhai Lu

Jinan University

Xinru Li

Shenzhen University

Juan Li

Jinan University

Liang Mao

China University of Mining and Technology

Xuandong Wang

Jinan University

Qin Chen

Jinan University https://orcid.org/0000-0001-8972-2568

long wen

Mingshan Zhu

Jinan University https://orcid.org/0000-0002-5926-5383

Baojun Li

Jinan University https://orcid.org/0000-0002-7481-7780

\section{Article}

Keywords: photocatalysis, nonmetallic plasmonic heterostructures, artificial photosynthesis

Posted Date: August 24th, 2021

DOI: https://doi.org/10.21203/rs.3.rs-822048/v1

License: (c) (i) This work is licensed under a Creative Commons Attribution 4.0 International License.

Read Full License 


\section{Abstract}

Constructing multi-physical effects on semiconductors is one new horizon to develop next-generation photocatalysts. Here we use pyroelectric black phosphorus (BP) to couple with nonmetallic plasmonic tungsten oxides (WO) forming a BP/WO heterostructures as photocatalysts to convert $\mathrm{CO}_{2}$ for $\mathrm{CO}$ under visible-near-infrared (Vis-NIR) light irradiation. Nonmetallic plasmonic heterostructures exhibit $26.1 \mu \mathrm{mol}$ $\mathrm{h}^{-1} \mathrm{~g}^{-1} \mathrm{CO}$ generation with a selectivity of $98 \%$, and which is 7 - and 17 -fold higher than those of plasmonic WO and pyroelectric BP, respectively. The interface P-O-W bonds in heterostructures are constructed to work as channels for electron transfer from BP to plasmonic WO. Moreover, the photothermal energy generated by SPR excitation on WO can make the temperature of heterostructures rapidly increasing from 24 to $86^{\circ} \mathrm{C}$ in 10 min, triggering the pyroelectric BP for carriers to promote electron transfer. Multi-physical effects including plasmonic hot carriers and photothermal effect of WO, intrinsic band excitation and pyroelectric effect of BP and W-O-P bonds play synergistic roles on boosting hot electron generation for $\mathrm{CO}_{2}$ reduction. This work provides clear proofs to demonstrate that constructing multi-physical effects on semiconductors is one useful strategy to promote NIR-harvesting for artificial photosynthesis.

\section{Introduction}

As the free carrier density reaches $10^{18}-10^{21} \mathrm{~cm}^{-2}$, the surface plasmon resonance (SPR) generally observed on noble metals also occurs on semiconductors such as $\mathrm{Cu}_{2-x} \mathrm{~S},{ }^{1,2} \mathrm{MoO}_{3-x} x^{3,4} \mathrm{WO}_{3-x}{ }^{5,6}$, and $\mathrm{Bi}_{2} \mathrm{O}_{3-\mathrm{x}}{ }^{7}$. Contrast to the metals ${ }^{8-10}$, nonmetallic plasmonic semiconductors have more complex electronic structures including intrinsic band gap, defect and SPR states, resulting in a complex excitation process and a broad light response in visible-near-infrared (Vis-NIR) region. ${ }^{11-14}$ Currently, the most reported semiconductors with Vis-NIR SPR absorption are focused on heavily oxygen-vacancy- or cationvacancy-doped metal oxides ${ }^{15,16}$, and the heavy doping is a valuable strategy to increase semiconductors' carrier density, resulting in their metalloid properties on optics and catalysis ${ }^{17-21}$. Taking plasmonic $\mathrm{WO}_{3-x}$ as an example ${ }^{22-24}$, the free-electron on heavy-oxygen-vacancy-doped $\mathrm{WO}_{3-\mathrm{x}}$ is excited by SPR to be hot electron for catalysis, meanwhile, the consumed electrons are supplied by the intrinsic band gap excitation or electron injection from other semiconductors for maintaining the free carrier density and SPR during photocatalysis ${ }^{11,22,23}$. However, the short lifetime of hot carriers ${ }^{22}$ on plasmonic semiconductors leads to fast nonradiative decay for photothermal energy, and it restricts the hot electron generation for photocatalysis. Therefore, the synergistic utilization of photothermal effect and hot carriers is one promising strategy to break the above limitations of plasmonic semiconductors for high-efficient photocatalysis.

The pyroelectric effect is one physical phenomenon to convert thermal energy for electrical energy, and which is triggered on crystal materials with non-centrosymmetric structures and has significant applications on pyroelectric nanosensors, electrical generators, and pyroelectric catalysis ${ }^{23-31}$. Recently, 
a pyroelectric-effect-induced electrical field was used to enhance photo-carriers separation in photocatalyst ${ }^{32,33]}$. A ternary heterostructure of poly (vinylidene fluoride-co-hexafluropropylene (PVDFHFP)/carbon nanotube (CNT)/CdS were reported with the triple functions of pyroelectric, photothermal material and photocatalyst, and in which the pyroelectric-effect-induced electrical field was demonstrated to promote carrier migration and separation for photocatalysis ${ }^{33}$. Therefore, constructing pyroelectric effect on plasmonic semiconductors will be a new direction for exploring high-efficient photocatalysts. Among various pyroelectric materials, two-dimensional (2D) layered black phosphorus (BP) with broken structure by the loss-of-inversion symmetry exhibits excellent pyroelectric effect and can realize pyro-catalytic $\mathrm{H}_{2}$ generation under cold-hot alternation ${ }^{34}$. Owning narrow band gap and pyroelectric effect ${ }^{[35-37]}, 2 \mathrm{D}$ BP is one ideal material to marry with plasmonic semiconductors forming heterostructures, and in which multi-physical effects may play synergistic roles on photocatalysis.

In this work, 2D structured BP with pyroelectric effect is coupled with plasmonic tungsten oxides $\left(\mathrm{WO}_{3}\right)$ to form heterostructures as photocatalysts for $\mathrm{CO}_{2}-\mathrm{RR}$. Under Vis-NIR irradiation, the nonmetallic plasmonic BP/WO heterostructures generate $26.1 \mu \mathrm{mol} \mathrm{h}^{-1} \mathrm{~g}^{-1} \mathrm{CO}$ with a selectivity of $98 \%$, and which is 7- and 17fold higher than those of plasmonic WO and pyroelectric BP, respectively. The interface W-O-P bonds to connect WO and BP are demonstrated by density functional theory (DFT) calculations and verified by various spectroscopic studies to work as electron transfer channels between BP and WO. Moreover, the pyroelectric effect of 2D BP can be triggered by photothermal energy from plasmonic WO for carriers to promote electron transfer. Therefore, multi-physical effects on BP/WO heterostructures including plasmonic photothermal effect and hot carriers of WO, intrinsic band excitation and pyroelectric effect of $\mathrm{BP}$ and W-O-P bonds play synergistic roles on boosting hot electron generation for high-selective $\mathrm{CO}$ generation. This work provides one useful strategy to promote photocatalytic performance of plasmonic semiconductors by constructing synergy of multi-physical effects on heterostructures.

\section{Results}

Samples characterization. Layered $\mathrm{BP}$ and $\mathrm{WO}_{3-\mathrm{x}}$ (WO) were synthesized by previous reported methods ${ }^{38,39}$, and their layer and nanowire morphologies were shown in TEM images (Supplementary Figs. 1a-d), respectively. HR-TEM images (Supplementary Figs. $1 \mathrm{~b}$ and $1 \mathrm{~d}$ ) show the 0.26 and $0.38 \mathrm{~nm}$ lattices spacing assigned to $\{040\}$ facets of $B P$ layers ${ }^{40}$ and $\{010\}$ facets of $\mathrm{W}_{18} \mathrm{O}_{49}$ nanowires ${ }^{41}$, respectively. BP/WO heterostructures were synthesized by using simple mixing in solution under ultrasonication, and TEM images (Figs. 1a and b) showed the structures of 10-BP/WO with 10 wt\% BP in composition. The 0.38 and $0.26 \mathrm{~nm}$ lattice spacing assigned to WO nanowires and layered BP are observed on HR-TEM image (Fig. 1c) of 10-BP/WO heterostructure. The constructed heterostructures were further characterized by the scanning TEM (STEM) coupled with energy-dispersive x-ray spectroscopy (EDX). The high-angle annular dark-field (HAADF) STEM image (Fig. 1d) shows the different contrast on the positions of nanowires and nanosheets in the heterostructures. Meanwhile, the distributions of $\mathrm{P}, \mathrm{O}$ and W elements are labeled in EDX mapping images (Fig. 1e-h). $O$ and W atoms are mainly located on nanowires, while, $\mathrm{P}$ atoms are located on nanosheets, verifying the BP/WO heterostructures. Crystal 
structures of BP/WO heterostructures were characterized by x-ray diffraction (XRD, Supplementary Fig. 2), and their XRD patterns are consistent with those of BP and WO without any other peaks.

UV-Vis-NIR diffuse reflectance spectra (DRS, Fig. 1i) shows the optical properties of different samples. 2D $\mathrm{BP}$ has a narrow band gap and a broad light response in visible region, and WO nanowires exhibit strong SPR absorption in Vis-NIR region. 10-BP/WO heterostructure has a similar SPR band with that of WO, and the slight enhancement UV-Vis absorption is attributed to $10 \mathrm{wt} \%$ BP loading. Chemical structures of 10BP/WO were analyzed by Raman spectra (Supplementary Fig. 3) and Fourier transform infrared (FTIR) spectra (Supplementary Fig. 4). Weak signal of P-O-W bonds was detected at $1116 \mathrm{~cm}^{-1}$, indicating that a small amount of P-O-W bonds exist on interface to connect $\mathrm{WO}$ and $\mathrm{BP} \mathrm{P}^{42,43}$. Oxygen vacancies in 10$\mathrm{BP} / \mathrm{WO}$ heterostrutures and plasmonic WO were detected by electron paramagnetic resonance (EPR) spectra and similar concentrations were observed in Fig. $1 \mathrm{j}^{[44]}$. X-ray photoelectron spectra (XPS) show the chemical state of elements in different samples. For BP, XPS of P 2p (Fig. 1k) are deconvoluted into two 129.5 and $130.4 \mathrm{eV}$ bands, assigned to $2 \mathrm{p}_{3 / 2}$ and $2 \mathrm{p}_{1 / 2}$, respectively ${ }^{45}$, and another bond at $133.5 \mathrm{eV}$ is attributed to the oxidized phosphorus $\left(P_{x} O_{y}\right)^{46}$. While, the bands of $P 2 p_{3 / 2}$ and $2 p_{1 / 2}$ on $10-B P / W O$ heterostructure have $0.5 \mathrm{eV}$ shift toward high binding energy direction, implying the decreased electronic affinity on BP. XPS of W $4 f$ on WO (Fig. 1l) show two broad bands which can be divided into four bands with binding energy of $35.1,37.1,36.1$ and $38.1 \mathrm{eV}$ assigned to $W^{5+} 4 f_{7 / 2}, W^{5+} 4 f_{5 / 2}, W^{6+} 4 f_{7 / 2}$, and $W^{6+}$ $4 f_{5 / 2}$, respectively ${ }^{47}$. The $W^{5+}$ is generated from the $W^{6+}$ reduction during the solvothermal process. For 10-BP/WO, W $4 \mathrm{f}$ bands have a $0.1 \mathrm{eV}$ shift toward low binding energy direction, showing the increased electron affinity on WO. Those results indicate the electrons transfer from BP to WO. P $=\mathrm{O}$ and $\mathrm{P}-\mathrm{OH}$ bonds in 10-BP/WO were detected by 0 1s XPS (Supplementary Fig. 5), implying the partial oxidation on layered $\mathrm{BP}$, and it provides the possible for BP to connect WO by W-O-P bonds.

The possiblity of charge transfer in BP/WO heterostructures were analyzed by the simulations. The density functional theory (DFT) was used to simulate charge interactions between $\mathrm{W}_{18} \mathrm{O}_{49}$ (001) surface and $2 \mathrm{D}$ BP to unravel the bonding mechanism. The charge density difference $\Delta \rho=\rho_{\mathrm{W}_{18} \mathrm{O}_{49}(001) / \mathrm{P}}-\rho_{\mathrm{W}_{18} \mathrm{O}_{49}(001)}-\rho_{\mathrm{P}}$ was calculated as shown in Fig. 2a. Results show that the charge density is redistributed by forming electron-rich and hole-rich regions at interfaces with small amount of $\mathrm{P}$ atoms bonded to $\mathrm{O}$ atoms. The electrons can be transferred from $\mathrm{P}$ to $\mathrm{W}_{18} \mathrm{O}_{49}(001)$ using an $\mathrm{O}$ bridge. To confirm our results, the work functions of the $\mathrm{W}_{18} \mathrm{O}_{49}(001)$ surface and black phosphorene were evaluated further. The vacuum levels $E_{\mathrm{v}}$ and Fermi levels $E_{\mathrm{f}}$ wereobtained as $E_{\mathrm{v}(\mathrm{W} 18049}$ $(001))=2.81 \mathrm{eV}, E_{\mathrm{v}(\mathrm{P})}=1.25 \mathrm{eV}, E_{\mathrm{f}(\mathrm{W} 18049(001))}=-2.80 \mathrm{eV}$, and $E_{\mathrm{f}(\mathrm{P})}=-3.84 \mathrm{eV}$. The work function $W$ can be obtained by $W=E_{\mathrm{V}}-E_{f}$, further we get $W_{\mathrm{W} 18049(001)}=5.61 \mathrm{eV}$ and $W_{(\mathrm{P})}=5.09 \mathrm{eV}$. The results quantitatively show that electrons can be transferred from $\mathrm{BP}$ to $\mathrm{W}_{18} \mathrm{O}_{49}$ (001) surface due to increasing work functions. The electron transfer process was verified by DRS, EPR and XPS spectroscopies, as shown in Figs. 2b-d. Under Vis-NIR irradiation, SPR band of 10-BP/WO becomes stronger with a blue shift as irradiation time prolongs, indicating the increasing electron density on plasmonic WO. The increasing electron density is also observed clearly on EPR spectra of 10-BP/WO after 15 min Vis-NIR irradiation. 
While, the DRS (Supplementary Fig. 6) and EPR spectra of plasmonic WO do not have changes under VisNIR-irradiation, confirming the electron transfer from BP to WO in BP/WO heterostructures. Moreover, the electron transfer frocess can also be observed on XPS of $W^{5+} 4 f$ (Fig. $2 d$ ) which have a pronounced increase on intensity after Vis-NIR irradiation, and the amount of $\mathrm{W}^{5+}$ is increased from 19.2 to $34.5 \mathrm{~mol} \%$ in heterostructures.

The kinetic process of electron transfer in different samples under light irradiation was characterized by using ultrafast transient absorption spectroscopy combined with the femtosecond laser excitation. As shown in transient absorption spectra of BP (Fig. 3a), the electron on VB of BP can be excited in a broad visible spectral region, and the highest photobleaching point is observed at $5.0 \mathrm{ps}$. Then, the excited electron has a decay resulting in a continuous decrease on spectra as time prolongs. At $2.0 \mathrm{~ns}, \Delta A$ is near zero meaning the completed decay of photoelectrons, and BP is recovered from an excited state to the ground state. So, the total lifetime of photoelectron on BP is about $2.0 \mathrm{~ns}$. While, for plasmonic WO, due to the short lifetime of plasmonic carriers ${ }^{48,49}$, the fast decay (<100 ps) process and weak signals on transient absorption spectra are observed in Fig. 3b. Therefore, the transient absorption spectra (Fig. 3c) of 10-BP/WO heterostructures are mainly from BP, and is much weaker than the spectra of pure BP. The photoelectron decay time (1.0 ns) in 10-BP/WO heterostructure is shorter than that in BP. More detailed photoelectron decay process was studied by the transient absorption kinetic curves (Fig. 3d) collected at $\lambda_{\text {pump }}=675 \mathrm{~nm}$. Two decay processes of direct decay $\left(\tau_{1}\right)$ with short time and trapped state decay $\left(\tau_{2}\right)$ with long time are simulated based on the kinetic curve. $\tau_{1}$ and $\tau_{2}$ are simulated to be $0.2 \mathrm{~ns}$ and $1.8 \mathrm{~ns}$ for $\mathrm{BP}$, and $0.16 \mathrm{~ns}$ and $1.3 \mathrm{~ns}$ for 10-BP/WO. The results indicate that a part of photoelectrons on BP directly transfers to $\mathrm{WO}$, leading to a fast decay process of photoelectrons on $\mathrm{BP}^{[50]}$. Consequently, the photo-excited photoelectrons on BP can transfer to plasmonic WO, and the continuous electron injection can increase the electron density for enhanced SPR.

Photocatalytic performance. The photocatalytic $\mathrm{CO}_{2}$ reduction reaction $\left(\mathrm{CO}_{2}-\mathrm{RR}\right)$ performance is determined in a reactor using a gas-solid configuration. Under Vis-NIR (>420 nm) light irradiation, 10BP/WO heterostructures generate $78.3 \mu \mathrm{mol} \mathrm{g}^{-1} \mathrm{CO}$ in 3 hours (Fig. 4a), more than 7- and 17-fold higher than 10.9 and $4.4 \mu \mathrm{mol} \mathrm{g}^{-1}$ of plasmonic WO and BP, respectively. CO generation rates (Supplementary Fig. 7) are calculated to be $26.1,3.6$, and $1.5 \mu \mathrm{mol} \mathrm{h}^{-1} \mathrm{~g}^{-1}$ with a selectivity of 98,97 , and $84 \%$ for 10 $\mathrm{BP} / \mathrm{WO}$, WO, and BP, respectively. Meanwhile, a small amount of $\mathrm{CH}_{4}$ is detected for the three samples (Supplementary Fig. 7). Different heterostructures with 2.5, 5.0, 7.5, 10, 12.5, and 15 wt.\% BP in composition were synthesized as photocatalysts, and 10-BP/WO exhibited the optimal performance on CO generation (Supplementary Fig. 8). CO generation rates for 2.5-BP/WO, 5.0-BP/WO, 7.5-BP/WO, 10BP/WO, 12.5-BP/WO, and 15-BP/WO are calculated to 7.3, 9.7, 19.1, 26.1, 20.5, and $11.3 \mu \mathrm{mol} \mathrm{h}^{-1} \mathrm{~g}^{-1}$ (Fig. 4b), with a selectivity of $91,95,95,98,98$, and $94 \%$, respectively. With pyroelectric effect and narrow band gap, the increased amount of BP will generate more pyroelectric electrons and photoelectrons to transfer to plasmonic WO which can enhance hot electron generation for $\mathrm{CO}_{2}-\mathrm{RR}$. However, as the amount of BP beyond $10 \mathrm{wt} \%$, the plasmonic WO nanowires are covered by BP layers which hinder the 
SPR excitation for photocatalytic $\mathrm{CO}_{2}$-RR. $\mathrm{CO}$ generated from $\mathrm{CO}_{2}$ is verified by using ${ }^{13} \mathrm{C}$-labled ${ }^{13} \mathrm{CO}_{2}$ for photocatalysis over $10-\mathrm{BP} / \mathrm{WO}$, and ${ }^{13} \mathrm{CO}$ are detected by the mass spectrometry (MS) as main product (Fig. 4c).

Considering the broad light absorption of 10-BP/WO heterostructures in UV-Vis-NIR region, their photocatalytic performance under different light irradiation was investigated as shown in Fig. 4d. UV- and Vis-irradiation only generates 7.2 and $7.9 \mu \mathrm{mol} \mathrm{g}^{-1} \mathrm{CO}$ in 3 hours, respectively. While, NIR-irradiation promotes $\mathrm{CO}$ generation to be $12.5 \mu \mathrm{mol} \mathrm{g}^{-1}$ in 3 hours. As Vis-NIR light is irradiated, CO generation is improved to $86.7 \mu \mathrm{mol} \mathrm{g}{ }^{-1}, 7$-fold higher than those under NIR irradiation. While, 109.4 and $148.5 \mu \mathrm{mol}$ $\mathrm{g}^{-1} \mathrm{CO}$ is generated under UV-Vis and full-spectrum (UV-Vis-NIR) light irradiation, respectively. CO generation rates (Fig. 4e) are calculated to 2.4, 2.6, 4.2, 36.5, 28.9, and $49.5 \mu \mathrm{mol} \mathrm{g}^{-1} \mathrm{~h}^{-1} \mathrm{CO}$ for UV, Vis, NIR, UV-Vis, Vis-NIR, and UV-Vis-NIR light irradiation, with a selectivity of 95, 94, 99, 100, 98, and $97 \%$, respectively. Only a littel $\mathrm{CH}_{4}\left(<1 \mu \mathrm{mol} \mathrm{g}{ }^{-1} \mathrm{~h}^{-1}\right)$ is detected during $\mathrm{CO}_{2}$-RR (Fig. 4e). The stability of 10$\mathrm{BP} / \mathrm{WO}$ heterostructures during photocatalytic $\mathrm{CO}_{2}-\mathrm{RR}$ were investigated as shown in Fig. $4 \mathrm{f}$. Light irradiation contianing UV can cause the decreased $\mathrm{CO}$ generation over 10-BP/WO, and CO generation rates are reduced from 49.5 to 17.8 and 36.5 to $8.9 \mu \mathrm{mol} \mathrm{g}^{-1} \mathrm{~h}^{-1}$ for UV-Vis-NIR- and UV-Vis-irradiation, respectively, during three times repeating $\mathrm{CO}_{2}-\mathrm{RR}$. The possible reason is the $\mathrm{BP}$ decompostion caused by UV-induced oxidation. Fortunately, 10-BP/WO heterostructures have a sustainable performance on CO generation during Vis-NIR-driven $\mathrm{CO}_{2}-\mathrm{RR}$. The chemical structure of 10-BP/WO after Vis-NIR-irradated photocatalysis is analyzed by XPS, and no obvious changes on P 2p and 01 s XPS (Supplementary Figs. 9 and 10) demonstrate their stable structures during $\mathrm{CO}_{2}$-RR. The apparent quantum efficiency (AQE) over different samples during $\mathrm{CO}_{2}$-RR was measured under monchrome light irradiation. AQE of 10BP/WO (Fig. 4g) has a similiar trend with SPR band confirming that photocatalytic $\mathrm{CO}$ generation is attributed to SPR excitation. More interesting is that AQE at $900-\mathrm{nm}$ is higher than those in visible region, and it means NIR-irradiation can enhance CO generation. However, AQE (Fig. 6h) of plasmonic WO shows a completely different result, much lower than that of 10-BP/WO in Vis-NIR region. A great difference on $\mathrm{AQE}$ indicates that pyroelectric $\mathrm{BP}$ can promote $\mathrm{CO}_{2}-\mathrm{RR}$ for high-selective $\mathrm{CO}$ generation. To enclude the influence of photoelectron on BP by Vis-excitation, NIR $(>800)$ light which can excite SPR of WO but weakly excite BP is used to irradiate BP, WO, 10-BP/WO for $\mathrm{CO}_{2}$-RR (Fig. 4i), and none, 1.1, and $4.2 \mu \mathrm{mol}$ $\mathrm{g}^{-1} \mathrm{~h}^{-1} \mathrm{CO}$ are generated, respectively. NIR-irradiation make SPR exciation to generate hot electrons and thermal energy simulatously on WO, and the thermal energy can trigger the pyroelectric BP for carriers, facliating electron transfer from $\mathrm{BP}$ to $\mathrm{WO}$ and hot electron generation for $\mathrm{CO}_{2}-\mathrm{RR}$.

The photothermal effect on 10-BP/WO was further investigated by the thermal camera. Thermal images of 10-BP/WO loaded glass plate (Supplementary Fig. 11) with different Vis-NIR light irradiation time are shown in Fig. 5a. Under Vis-NIR light irradiation, the temperature on 10-BP/WO have a rapidly increase from initial $24.3^{\circ} \mathrm{C}$ to $57.1^{\circ} \mathrm{C}$ in $2 \mathrm{~min}$, and then to $70.8^{\circ} \mathrm{C}$ in $4 \mathrm{~min}$. Finally, the temperature is stablized at $86^{\circ} \mathrm{C}$ after $10 \mathrm{~min}$ irradiation. Thermal images and temperatures of 10-BP/WO under different light irradiation are measured in Fig. 5b and Supplementary Figs. 12-16. The temperature is stablized at 42.5, 
$62.2,83,66.5,86.3$ and $98.5^{\circ} \mathrm{C}$ with the $\Delta \mathrm{T}$ of $15.6,37.2,59.7,40.5,62.0$ and $72.2^{\circ} \mathrm{C}$ under $10 \mathrm{~min} \mathrm{UV}$, Vis, NIR, UV-Vis, Vis-NIR and UV-Vis-NIR light irradiation, respectively. It is clear that the main contribution on photothermal energy of 10-BP/WO comes from NIR-irradiation, attributing to the strong SPR absorption in NIR region. The photothermal energy generated on plasmonic WO can trigger the pyroelectric BP of heterostructures for carriers and pyroelectric field faciliating the electron transfer to plasmonic WO for $\mathrm{CO}_{2}-\mathrm{RR}$. To verify the contribution of pyroelectric effect on photocatalysis, $\mathrm{CO}_{2}-\mathrm{RR}$ under heating $\left(85^{\circ} \mathrm{C}\right)$, Vis-irradiation $(420-780 \mathrm{~nm})$ and heating/Vis-irradiation over 10-BP/WO were tested as shown in Fig. $5 \mathrm{c}$, and $1.4,7.9$ and $13.5 \mu \mathrm{mol} \mathrm{g}{ }^{-1} \mathrm{CO}$ were obtained in 3 hours, respectively. Only heating on 10-BP/WO do not improve $\mathrm{CO}_{2}-\mathrm{RR}$. However, heating can greatly enhance Vis-driven $\mathrm{CO}_{2}-\mathrm{RR}$ on 10-BP/WO. The possible illustration is that the heating triggers the pyroelectric $\mathrm{BP}$ to convert thermal energy for carriers and transfer to WO promoting plasmonic $\mathrm{CO}_{2}-\mathrm{RR}$. The influence of heating on $\mathrm{CO}_{2}-\mathrm{RR}$ over WO and BP as catalysts were also tested in Fig. $5 \mathrm{~d}$, and the heating and Vis-irradiation do not play synergistic roles on $\mathrm{CO}_{2}-\mathrm{RR}$. Consequently, SPR and pyrolectric effect in BP/WO heterostructures play an synergistic role on Vis snd NIR light harvesritng for continnous eletron injection and hot electron generation for high-selective $\mathrm{CO}$ generation.

The detailed reaction pathway during $\mathrm{CO}_{2}$-RR over BP/WO was monitored by in situ FTIR spectra, as shown in Fig. 6a. Before $\mathrm{CO}_{2}$ absorption in the dark, no remarkable signals are observed on the spectra. After $\mathrm{CO}_{2}$ saturated absorption for $30 \mathrm{~min}$ in the dark, the bands of bidentate carbonate $\left(\mathrm{b}-\mathrm{CO}_{3}{ }^{2-}, 1635\right.$ $\left.\mathrm{cm}^{-1}\right)$, monodentate carbonate $\left(\mathrm{m}-\mathrm{CO}_{3}{ }^{2-}, 1507 \mathrm{~cm}^{-1}\right)$, and bicarbonate $\left(\mathrm{HCO}_{3}{ }^{-}, 1416 \mathrm{~cm}^{-1}\right)$ are detected on the surface of $10-\mathrm{BP} / \mathrm{WO}^{51,52}$. When Vis-NIR light is irradiated, the intensity of b- $\mathrm{CO}_{3}{ }^{2}$ bonds has a increase as time prolongs, implying $\mathrm{b}-\mathrm{CO}_{3}{ }^{2}$ as dominant absorption mode on surface. Moreover, the bicarbonate $\mathrm{HCO}_{3}{ }^{-}$bands become stronger implying that $\mathrm{HCO}_{3}{ }^{-}$is intermediate of $\mathrm{CO}_{2}$ reduction. So, the possible reaction pathway of $\mathrm{CO}$ generation is that $\mathrm{CO}_{2}$ adsorbed on WO surface forms b- $\mathrm{CO}_{3}{ }^{2-}$, and then reacts with hot electron and a proton to form intermediate $\mathrm{HCO}_{3}{ }^{-} . \mathrm{HCO}_{3}{ }^{-}$further reacts with electrons and protons to form $\mathrm{CO}$ and $\mathrm{H}_{2} \mathrm{O}$. No absorbed ${ }^{*} \mathrm{CO}$ is detected by in situ FTIR spectra, indicating the fast desorption of $\mathrm{CO}$ as product. The DFT calculations were used to simulate the detailed reaction pathway of $\mathrm{CO}_{2}-\mathrm{RR}$. The Gibbs reaction free energy change $(\Delta \mathrm{G})$ during $\mathrm{CO}_{2}$ reduction over $\mathrm{WO}_{3-\mathrm{x}}$ was calculated as shown in Fig. $6 \mathrm{~b} . \mathrm{CO}_{2}$ is absorbed on the surface of $\mathrm{WO}_{3-\mathrm{x}}$ to be ${ }^{*} \mathrm{CO}_{2}$ with $\Delta \mathrm{G}=0.35 \mathrm{eV}$. Then, one electron and proton are obtained by ${ }^{*} \mathrm{CO}_{2}$ to be ${ }^{*} \mathrm{COOH}$ with $\Delta \mathrm{G}=-0.53 \mathrm{eV}$. After reaction with the second electron and proton, ${ }^{*} \mathrm{COOH}$ becomes ${ }^{*} \mathrm{CO}$ with a $\Delta \mathrm{G}=-0.45 \mathrm{eV}$. ${ }^{*} \mathrm{CO}$ reaction for next step has two different pathways, one is to desorb from surface to be free $\mathrm{CO}$ with a $\Delta \mathrm{G}=0.65 \mathrm{eV}$, and the other one is to reaction with one electron and proton to be ${ }^{*} \mathrm{CHO}$ with a $\Delta \mathrm{G}=0.91 \mathrm{eV}$ which is generally considered as a key step for $\mathrm{CH}_{4}$ generation. In situ FITR and DFT simulations demonstrate that $\mathrm{CO}$ generation is the main reaction pathway of $\mathrm{CO}_{2}$-RR over plasmonic WO as photocatalyst, and it is consistent with the experiment results. 
Based on above results, the detailed multi-synergetic processes and the possible photocatalytic mechanism are described in schematic diagram (Fig. 6c). With abundant oxygen vacancies, a defect band (DB) formed on the bottom of the conduction band (CB) in WO nanowires. With abundent $\mathrm{W}^{5+}$, the DB is occupied by the electrons generating SPR on WO in Vis-NIR region, and the large energy difference between valence band (VB) and lowest unoccupied CB restricts electron excitation from VB to CB under Vis-NIR irradiation. While, the electrons on DB can be excited by SPR to be hot electron in WO. The fast decay process of hot carriers leads to short lifetime of hot electron ( $<200 \mathrm{ps})$ which has been observed on transient absorption spectra (Fig. 4). The nonradiative decay of hot carriers generates photothermal energy causing the low efficiency of plasmonic photocatalysis. Combination of pyrolectric BP and plasmonic WO forms heterostructures, and the plasmonic photothermal energy on WO nanowires can transfer to BP by the thermal radiation, triggering pyroelectric field for carriers on BP. Moreover, the visible irradiation on BP also generates photo-carriers. For plasmonic WO, SPR-excitation can generate hot electrons at high energy levels which are acitve for photocatalysis. W-O-P bonds connected WO and BP are demonstrated to be channels for electron transfer (ET) from BP to WO. Therefore, the electrons generated by photo-excitation and pyroelectric effects on CB of BP can both transfer to DB of WO with low energy level. The continuous electron injection from BP to plasmonic WO will increase the electron density on DB for stronger SPR which can generate more active hot electrons. Moreover, the continuous electron injection restricts hot electron deacy from high energy states to low energy states, promoting hot eletrons for $\mathrm{CO}_{2}$ reduction. Meanwhile, the holes left on VB of BP have an oxidization reaction. Under UVirradiation, electron on VB of WO can be excited to DB of WO leading to the increased electron density for stronger SPR, and it is favor for plasmonic $\mathrm{CO}_{2}$-RR. Meanwhile, the holes left on VB of WO are active to decompose $\mathrm{BP}$, causing the unsustainable activity of heterostructures on photocatalytic $\mathrm{CO}_{2}-\mathrm{RR}$. Consequently, multi-physical effects including plasmonic hot carriers and photothermal effect of WO, pyrolectric effect and band excitation of BP, and electron transfer through W-O-P bonds play synergetic roles on enhancing SPR and hot electron generation, boosting high-selective $\mathrm{CO}_{2}-\mathrm{RR}$ for $\mathrm{CO}$ generation.

\section{Discussion}

In summary, a pyroelectric/plasmonic BP/WO heterostructure was constructed as photocatalysts with multi-physical effects for $\mathrm{CO}_{2}$ reduction. $26.1 \mu \mathrm{mol} \mathrm{h} \mathrm{h}^{-1} \mathrm{~g}^{-1} \mathrm{CO}$ was generated over the nonmetallic plasmonic heterostructures under Vis-NIR light irradiation with a selectivity of $98 \%$, and which was 7 - and 17-fold higher than those of plasmonic WO and BP, respectively. W-O-P bonds connected WO and BP were demonstrated by DFT calculations and varified by various spectrascopic studies as channels for electron transfer from BP to WO. The photothermal energy generated by SPR of WO can make temperature of 10BP/WO rapidly increase from 24 to $86^{\circ} \mathrm{C}$ under Vis-NIR light irradiation, triggering the pyroelectric effect of BP for carriers to promote electron transfer. Therefore, multi-synergistic effects of plasmonic hot carriers and photothermal effect on WO, band excitation and pyroelectric effects on BP, and electron transfer through W-O-P bonds promote hot electron generation for high-selective $\mathrm{CO}_{2}$-RR in Vis-NIR region. 
Our work provids clear proofs to demonstrate that contructing multi-physical effects on semiconductors are one promising strategy to improve the Vis-NIR light haversting for artifical photosynthesis.

\section{Methods}

Materials. Tungsten chloride $\left(\mathrm{WCl}_{6},>99 \%\right)$ was obtained from Sigma-Aldrich, ethanol (99.8\%) was obtained from Macklin chemical reagent Corp, N, N dimethyl formamide (DMF, Aladdin), N-Methyl pyrrolidine (NMP, AR, Aladdin), carbon dioxides (>99.999\%) was obtained Dalian Special Gases Co., LTD, Milli-Q water (18.2 M $\Omega$ ) was obtained from Sartorius. High-quallity of black phosphorus (BP) was purchased from Zhengzhou ADD Intelligent Technology Co., Ltd. (ADDC1911017, www.addtech.net.cn). All the chemical reagents were used as purchased without any further purification.

Sample preparation. Layered BP were obtained by using a basic NMP solvent exfoliation method. In detail, $20 \mathrm{mg}$ of bulk BP was first dispersed in $20 \mathrm{~mL}$ NMP, and then the dispersion was sonicated for $4 \mathrm{~h}$ at $100 \mathrm{~W}$ output power under ice-water bath. After exfoliation, the dispersion was centrifuged at $2000 \mathrm{rpm}$ for 20 min with two times to remove non-exfoliated bulk BP. The supernatant was further centrifuged at $11,000 \mathrm{rpm}$ for $20 \mathrm{~min}$ to separate BP from the supernatant. After that, the precipitations were redispersed in $10 \mathrm{~mL}$ NMP with ultrasonic process, resulting in BP NMP dispersion $\left(0.5 \mathrm{mg} \mathrm{mL}^{-1}\right)$.

$\mathrm{WO}_{3-\mathrm{x}}$ (WO) nanowires were obtained by using solvothermal method. In a typical procedure, $150 \mathrm{mg}$ of $\mathrm{WCl}_{6}$ powder was dissolved into $30 \mathrm{~mL}$ ethanol, which was vigorously stirred to obtain a yellow suspension. Subsequently, the solution was transferred to a $50 \mathrm{~mL}$ Teflon-lined stainless steel autoclave, heated and maintained at $180^{\circ} \mathrm{C}$ for $24 \mathrm{~h}$. After naturally cooling down to room temperature, the dark blue powder was separated by centrifugation, washed with ethanol for three times and dried in a vacuum oven for overnight.

BP/WO heterostructures were obtained by a simple ultrasonic process. In brief, BP/NMP solution was centrifuged and washed with ethanol for twice to remove the NMP. Subsequently, the $10 \mathrm{mg}$ WO was dispersed $10 \mathrm{~mL}$ ethanol with sonicated for $15 \mathrm{~min}$, then the $2 \mathrm{~mL}$ as-prepared BP/ethanol dispersion (0.5 $\mathrm{mg} \mathrm{mL}^{-1}$ ) was added into above WO dispersion with sonication for $5 \mathrm{~min}$. Then, the BP/WO were precipitated and obtained by centrifugation. The obtained BP/WO sample was labelled as 10-WO/BP and the different weight ratio BP for WO were prepared by adding different amounts of BP to WO.

Samples characterization. The X-ray diffraction (XRD) patterns of the sample were carried out by a Rigaku Rint-2500 diffractometer with $\mathrm{Cu} \mathrm{K}_{\mathrm{a}}$ radiation at a scanning rate of $0.1^{\circ} \mathrm{s}^{-1}$. The morphologies were measured by transmission electron microscope (2100, JEOL, operated at $100 \mathrm{kV})$. X-ray photoelectron spectroscopy (XPS) measurements were performed at a Thermo Fisher Scientific K-ALPHA + spectrometer. The binding energy was referenced to the $\mathrm{C} 1 \mathrm{~s}$ peak at $284.6 \mathrm{eV}$ of the adventitious carbon. Electron paramagnetic resonance (EPR) signal were detected by Bruker A300 spectrometer. UVVis-NIR diffuse reflectance spectra (UV-Vis-NIR DRS) were recorded in a UV-Vis/NIR spectrophotometer (JASO V-570). Raman spectra were obtained on a Raman microscopy (XPLORA PLUS, HORIBA) with a 
532-nm laser for excitation. Fourier transform infrared (FTIR) spectra were collected by a spectrometer (THERMO) employing KBr disk method. The in situ FTIR spectra were obtained through an in situ diffuse reflectance Fourier transform infrared spectrometer (Nicolet iS50, TMO, US). The sample was degassed for $4 \mathrm{~h}$ at $150^{\circ} \mathrm{C}$ prior to measurement. Then each sample was purged with nitrogen for one hour to blow out all the gases adsorbed on the samples. After that, a mixed gas of $\mathrm{CO}_{2}$ and water vapor was flowed into the specimen chamber for another hour to ensure sorption equilibrium before irradiation.

Photocatalytic $\mathrm{CO}_{2}$ reduction test. $5 \mathrm{mg}$ sample was mixed with $0.2 \mathrm{~mL}$ pure water and plastered on cover glass $\left(4.9 \mathrm{~cm}^{-2}\right)$. The cover glass with the sample on the upside was put on the bottom of the reaction chamber $(100 \mathrm{~mL})$. The chamber was sealed with thick quartz cover glass and degassed with pure carbon dioxide gas for $20 \mathrm{~min}$. Subsequently, Vis-NIR light supplied by a $300 \mathrm{~W}$ xenon lamp (Perfectlight, PLS-SXE300D) equipped with a wavelength cutoff filter ( $>400 \mathrm{~nm})$ irradiated the suspension. The gaseous products were analysed by gas chromatography (GC-2014A, Shimadzu) equipped with one TCD and two flame ionization detectors (FID). Other photocatalytic reaction measurements were carried following the above procedure under different light irradiation which is supplied by a $300 \mathrm{~W}$ xenon lamp equipped with different-wavelength cutoff filters $(<400 \mathrm{~nm}, 400-780$ $\mathrm{nm},>800 \mathrm{~nm})$.

$\mathrm{CO}_{2}$ photoreduction under monochromatic light using the different monochromatic filters provided the apparent quantum efficiency (AQE). An optical power meter (FZ-A) measured the light intensities. The $A Q E$ was calculated based on the following equation: $A Q E \%=(N \times$ Number of evolved production molecules)/ (Number of incident photons) $\times 100 \%$.

Density functional theory (DFT) calculations. All density functional theory (DFT) calculations are performed using the using the Vienna Ab-initio Simulation Package (VASP) software ${ }^{53,54}$ The exchangecorrelation functional is approximated by using the generalized gradient approximation (GGA) ${ }^{55}$ with Perdew-Burke-Ernzerhof (PBE) ${ }^{56}$ rametrization. The cutoff energy of the plane-wave is set to be $500 \mathrm{eV}$ to ensure convergence. The convergence criteria of the force and the energy are $10^{-3} \mathrm{eV} / \AA$ and $10^{-7} \mathrm{eV}$, respectively. The $2 \mathrm{D}$ Brillouin zone integration is done with a k-mesh density of $60 / a^{57}$ where a denotes the length of the lattice constant in the unit of $\AA$. In the slab model, the vacuum region is set as $20 \AA$ to avoid interactions between adjacent layers. The $\mathrm{W}_{18} \mathrm{O}_{49}(001)$ surface was modeled from the $\mathrm{W}_{18} \mathrm{O}_{49}$ bulk unit cell with the optimized lattice parameters of $a=3.859 \AA$ and $b=18.287 \AA$. The black phosphorene was modelled with the optimized lattice parameters of $a=4.626 \AA$ and $b=3.298 \AA$. To simulate the heterostructure $\mathrm{W}_{18} \mathrm{O}_{49}(001)$ / black phosphorene, a supercell $4 \times 1$ of black phosphorene is used to match the $1 \times 1 \mathrm{~W}_{18} \mathrm{O}_{49}(001)$ with the optimized lattice parameters of $\mathrm{a}=4.626 \AA$ and $\mathrm{b}=3.298$ $\AA$. Due to the absence of strong bonding interactions between $\mathrm{W}_{18} \mathrm{O}_{49}(001)$ surface and black phosphorene, the PBE that forms with vdW correction (PBE-D2) is chosen due to the good description of long-range vdW interaction ${ }^{58}$. 
The free energy computations are performed by using the slab model. A vacuum of approximately $15 \AA$ is placed above the slabs to separate the interaction between periodic images. For $\mathrm{W}_{18} \mathrm{O}_{49}$ (001) surface slab model, a $1 \times 1$ twice atomic layer including $98 \mathrm{~S}$ atoms and $36 \mathrm{~W}$ atoms is used. The free energy of the adsorbed state is calculated based on the adsorption energy as follows:

$\Delta \mathrm{Gads}=$ Eads $+\Delta \mathrm{EZPE}-\mathrm{T} \Delta \mathrm{S}$,

where Eads is the adsorption energy, and $\triangle E Z P E$ is the difference corresponding to the zero-point energy between the adsorbed state and the gas phase, $S$ is the entropy, and $T$ is the temperature.

\section{Declarations}

\section{Competing interests $\square$}

The authors declare no competing interests.

\section{Additional information}

Supplementary information is available for this parper on the website.

\section{References}

1. Zhou, L.; Liu, Z. H.; Guan, Z. P.; Tian, B. Z.; Wang, L. Z.; Zhou, Y.; Zhou, Y. B.; Lei, J. Y.; Zhang, J. L.; Liu, Y. D. OD/2D Plasmonic $\mathrm{Cu}_{2}-{ }_{\mathrm{x}} \mathrm{S} / \mathrm{g}-\mathrm{C}_{3} \mathrm{~N}_{4}$ Nanosheets Harnessing UV-vis-NIR Broad Spectrum for Photocatalytic Degradation of Antibiotic Pollutant. Appl. Catal B-Environ. 263, 118326 (2020).

2. Liu, Z.; Liu, X. j.; Du, Y. D.; Ren, J. S.; Qu, X. G. Using Plasmonic Copper Sulfide Nanocrystals as Smart Light-Driven Sterilants. ACS Nano, 9, 10335-1034 (2015).

3. Li, J.; Ye, Y. H.; Ye, L. Q.; Su, F. Y.; Ma, Z. Y.; Huang, J. D.; Xie, H. Q.; Doronkin, D. E.; Zimina, A.; Grunwaldt, J. D.; Zhou, Y. Sunlight Induced Photo-Thermal Synergistic Catalytic $\mathrm{CO}_{2}$ Conversion via Localized Surface Plasmon Resonance of $\mathrm{MoO}_{3}$ - x. J. Mater. Chem. A, 7, 2821-2830 (2019).

4. Cheng, H. F.; Qian, X. F.; Kuwahara, Y.; Mori, K.; Yamashita, H. A Plasmonic Molybdenum Oxide Hybrid with Reversible Tunability for Visible-Light-Enhanced Catalytic Reactions. Adv. Mater. 27, 4616-4621 (2015).

5. Manthiram, K.; Alivisatos, A. P. Tunable Localized Surface Plasmon Resonances in Tungsten Oxide Nanocrystals. J. Am. Chem. Soc. 134, 3995-3998 (2012).

6. Li, J.; Chen, G. Y.; Yan, J. H.; Huang, B. B.; Cheng, H. F.; Lou, Z. Z.; Li, B. J. Solar-Driven Plasmonic Tungsten Oxides as Catalyst Enhancing Ethanol Dehydration for Highly Selective Ethylene Production. Appl. Catal. B-Environ., 264, 118517 (2020).

7. Li, Y. X.; Wen, M. M.; Wang, Y.; Tian, G.; Wang, C. Y.; Zhao, J. C. Plasmonic Hot Electrons from Oxygen Vacancies for Infrared Light-Driven Catalytic $\mathrm{CO}_{2}$ Reduction on $\mathrm{Bi}_{2} \mathrm{O}_{3}-\mathrm{x}$. Angew. Chem. Int. Ed. 60, 910-916 (2021). 
8. Chen, W.; Li, X. J.; Wang, F.; Javaid, S.; Pang, Y. P.; Chen, J. Y.; Yin, Z. Y.; Wang, S. B.; Li, Y. G.; Jia, G. H. Nonepitaxial Gold-Tipped ZnSe Hybrid Nanorods for Efficient Photocatalytic Hydrogen Production. Small 16, 1902231 (2019).

9. Bian, Z. F.; Tachikawa, T.; Zhang, P.; Fujitsuka, M.; Majima, T. Au/TiO 2 Superstructure-Based Plasmonic Photocatalysts Exhibiting Effıcient Charge Separation and Unprecedented Activity. J. Am. Chem. Soc. 136, 456-465 (2014).

10. Vu, N. N.; Kaliaguine, S.; Do, T. O. Plasmonic Photocatalysts for Sunlight-Driven Reduction of $\mathrm{CO}_{2}$ : Details, Developments, and Perspectives. ChemSusChem, 13, 3967-3991 (2020).

11. Lu, C. H.; Li, J.; Yan, J. H.; Li, B. J.; Huang, B. B.; Lou, Z. Z. Surface Plasmon Resonance and Defects on Tungsten Oxides Synergistically Boost High-Selective $\mathrm{CO}_{2}$ Reduction for Ethylene. Appl. Mater. Today, 20, 100744 (2020).

12. Lu, C. H.; Li, X. R.; Wu, Q.; Li, J.; Wen, L.; Dai, Y.; Huang, B. B.; Li, B. J.; Lou, Z. Z. Constructing Surface Plasmon Resonance on $\mathrm{Bi}_{2} \mathrm{WO}_{6}$ to Boost High-Selective $\mathrm{CO}_{2}$ Reduction for Methane. ACS Nano, 15, 3529-3539 (2021).

13. Li, J.; Xu, X. H.; Huang, B. B.; Lou, Z. Z.; Li, B. J. Light-Induced in Situ Formation of a Nonmetallic Plasmonic $\mathrm{MoS}_{2} / \mathrm{MoO}_{3-\mathrm{x}}$ Heterostructure with Efficient Charge Transfer for $\mathrm{CO}_{2}$ Reduction and SERS Detection. ACS Appl. Mater. Interfaces, 13, 10047-10053 (2021).

14. Li, J.; Lou, Z. Z.; Li, B. J. Nanostructured materials with localized surface plasmon resonance for photocatalysis. Chinese. Chem. Lett. DOI: 10.1016/j.cclet.2021.07.059 (2021)

15. Yin, H. B.; Kuwahara, Y.; Mori, K.; Yamashita, H. Plasmonic Metal/MoxW $\mathrm{M}_{1-\mathrm{x}} \mathrm{O}_{3-\mathrm{y}}$ for Visible-LightEnhanced $\mathrm{H}_{2}$ Production from Ammonia Borane. J. Mater. Chem. A, 6, 10932-10938 (2018).

16. Le, H. K. D.; Xiong, H. Y.; Page, B. A.; Garacia-Herrera, L. F.; McAllister, H. P.; Li, B. C.; Wang, H. Y.; Plass, K. E. Effects of $\mathrm{I}_{2}$ on $\mathrm{Cu}_{2-\mathrm{x}} \mathrm{S}$ Nanoparticles: Enabling Cation Exchange but Complicating Plasmonics. ACS Materials Lett. 2, 140-146 (2020).

17. Greenberg, B. L.; Ganguly, S.; Held, J. T.; Kramer, N. J.; Mkhoyan, K. A.; Aydil, E. S.; Kortshagen, U. R. Nonequilibrium-Plasma-Synthesized ZnO Nanocrystals with Plasmon Resonance Tunable via Al Doping and Quantum Confinement. Nano Lett. 15, 8162-8169 (2015).

18. Zandi, O.; Agrawal, A.; Shearer, A. B.; Reimnitz, L. C.; Dahlman, C. J.; Staller, C. M.; Milliron, D. J. Milliron. Impacts of Surface Depletion on the Plasmonic Properties of Doped Semiconductor Nanocrystals. Nat. Mater. 17, 710-717 (2018).

19. Tandon, B.; Yadav, A.; Khurana, D.; Reddy, P.; Santra, P. K.; Nag, A. Size-Induced Enhancement of Carrier Density, LSPR Quality Factor, and Carrier Mobility in Cr-Sn Doped $\ln _{2} \mathrm{O}_{3}$ Nanocrystals. Chem. Mater. 29, 9360-9368 (2017).

20. Bhattacharya, C.; Saji, S. E.; Mohan, A.; Madav, V.; Jia, G. H.; Yin, Z. Y. Sustainable NanoplasmonEnhanced Photoredox Reactions: Synthesis, Characterization, and Applications. Adv. Energy Mater. 10, 2002402 (2020). 
21. Li, J.; Lou, Z. Z.; Li, B. J. Engineering plasmonic semiconductors for enhanced photocatalysis. J. Mater. Chem. A DOI: 10.1039/d1ta04541e (2021)

22. Lu, C. H.; Li, J.; Chen, G. Y.; Li, B. J.; Lou, Z. Z. Self-Z-scheme plasmonic tungsten oxide nanowires for boosting ethanol dehydrogenation under UV-visible light irradiation. Nanoscale 11, 12774-12780 (2019).

23. Lou, Z. Z.; Zhang, P.; Li, J.; Yang, X. G.; Huang, B. B.; Li, B. J. Plasmonic Heterostructure $\mathrm{TiO}_{2}{ }^{-}$ $\mathrm{MCs} / \mathrm{WO}_{3}-\mathrm{x}$-NWs with Continuous Photoelectron Injection Boosting Hot Electron for Methane Generation. Adv. Funct. Mater. 29, 1808696 (2019).

24. Liu, Z.; Ren, W. J.; Peng, P.; Guo, S. B.; Lu, T.; Liu, Y.; Dong, X. L.; Wang, G. S. High Performance $\mathrm{Bi}_{0.5} \mathrm{Na}_{0.5} \mathrm{TiO}_{3}-\mathrm{BiAlO}_{3}-\mathrm{K}_{0.5} \mathrm{Na}_{0.5} \mathrm{NbO}_{3}$ Lead-Free Pyroelectric Ceramics for Thermal Detectors. Appl. Phys. Lett. 112, 142903 (2018).

25. Suen, J. Y.; Fan, K.; Montoya, J.; Bingham, C.; Stenger, V.; Sriram, S.; Padilla, W. J. Multifunctional Metamaterial Pyroelectric Infrared Detectors. Optica 4, 276-279 (2017).

26. Bowen, C. R.; Taylor, J.; LeBoulbar, E.; Zabek, D.; Chauhanc, A.; Vaish, R. Pyroelectric Materials and Devices for Energy Harvesting Applications. Energ. Environ. Sci. 7, 3836-3856 (2014).

27. Wang, Z.; Yu, R.; Pan, C.; Li, Z. L.; Yang, J.; Yi, F.; Wang, Z. L. Light-Induced Pyroelectric Effect as an Effective Approach for Ultrafast Ultraviolet Nanosensing. Nat. Commun. 6, 8401 (2015).

28. Yang, Y.; Zhou, Y. S.; Wu, J. M.; Wang, Z. L. Single Micro/Nanowire Pyroelectric Nanogenerators as Self-Powered Temperature Sensors. ACS Nano 6, 8456-8461 (2012).

29. Wang, X. F.; Dai, Y. J.; Liu, R. Y.; He, X. Li, S. Wang, Z. L. Light-Triggered Pyroelectric Nanogenerator Based on a pn-Junction for Self-Powered Near-Infrared Photosensing. ACS Nano 11, 8339-8345 (2017).

30. Su, R.; Shen, Y.; Li, L.; Zhang, D.; Yang, G.; Gao, C.; Yang, Y. Silver-Modified Nanosized Ferroelectrics as a Novel Photocatalyst. Small 11, 202-207 (2015).

31. Liu, G.; Ma, L.; Yin, L. C.; Wan, G.; Zhu, H.; Zhen, C.; Yang, Y.; Liang, Y.; Tan, J.; Cheng, H. M. Selective Chemical Epitaxial Growth of $\mathrm{TiO}_{2}$ Islands on Ferroelectric $\mathrm{PbTiO}_{3}$ Crystals to Boost Photocatalytic Activity. Joule 2, 1095-1107 (2018).

32. Li, J.; Cai, L.; Shang, J.; Yu, Y.; Zhang, L. Giant Enhancement of Internal Electric Field Boosting Bulk Charge Separation for Photocatalysis. Adv. Mater. 28, 4059-4064 (2016).

33. Dai, B. Y.; Fang, J. J.; Yu, Y. R.; Sun, M. L.; Huang, H. M.; Lu, C. H.; Kou, J. H.; Zhao, Y. J.; Xu, Z. Z. Construction of Infrared-Light-Responsive Photoinduced Carriers Driver for Enhanced Photocatalytic Hydrogen Evolution. Adv. Mater. 32, 1906361 (2020).

34. You, H. L.; Jia, Y.; Wu, Z.; Wang, F. F.; Huang, H. T.; Wang, Y. Room-Temperature Pyro-Catalytic Hydrogen Generation of 2D Few-Layer Black Phosphorene under Cold-Hot Alternation. Nat. Comm. 9, 2889 (2018).

35. Tian, B.; Tian, B.; Smith, B.; Scott, M. C.; Hua, R.; Lei, Q.; Tian, Y. Supported black phosphorus nanosheets as hydrogen-evolving photocatalyst achieving $5.4 \%$ energy conversion efficiency at 353 
K. Nat. Commun. 9. 1397 (2018).

36. Wu, Z. H.; Lyu, Y.; Zhang, Y.; Ding, R.; Zhang, B.; Yang, Z. B.; Lau, S. P.; Chen, X. H.; Hao, J. H. Largescale growth of few-layer two-dimensional black phosphorus. Nat. Mater. 15, 6146 (2021).

37. Zhu, X. J.; Zhang, T.; Jiang, D. C.; Duan, H.; Sun, Z.; Zhang, M. M.; Jin, H. C.; Guan, R.; Liu, Y. J.; Chen, M. Q.; Ji, H. X.; Du, P.; Yan, W. S.; Wei, S. Q.; Lu, Y. L.; Yang, S. F. Stabilizing black phosphorus nanosheets via edge-selective bonding of sacrificial $\mathrm{C}_{60}$ molecules. Nat. Commun. 9, 4177 (2018)

38. Wang, X. D.; He, J.; Li, J. Y.; Lu, G.; Dong, F.; Majima, T.; Zhu, M. S. Immobilizing Perovskite $\mathrm{CsPbBr}_{3}$ Nanocrystals on Black Phosphorus Nanosheets for Boosting Charge Separation and Photocatalytic $\mathrm{CO}_{2}$ Reduction. Appl. Catal B-Environ. 277, 119230 (2020).

39. Xi, G. C.; Ouyang, S. X.; Li, P.; Ye, J. H.; Ma, Q.; Su, N.; Bai, H.; Wang, C. Ultrathin $W_{18} \mathrm{O}_{49}$ Nanowires with Diameters below $1 \mathrm{~nm}$ : Synthesis, Near-Infrared Absorption, Photoluminescence, and Photochemical Reduction of Carbon Dioxide. Angew. Chem. Int. Ed. 51, 2395-2399 (2012).

40. Zhu, M. S.; Kim, S.; Mao, L.; Fujitsuka, M.; Zhang, J. Y.; Wang, X. C.; Majima, T. Metal-Free Photocatalyst for $\mathrm{H}_{2}$ Evolution in Visible to Near-Infrared Region: Black Phosphorus/Graphitic Carbon Nitride. J. Am. Chem. Soc. 139, 13234-13242 (2017).

41. Wang, Y.; Wang, X. X.; Xu, Y. H.; Chen, T.; Liu, M. L.; Niu, F. S.; Wei, S.; Liu, J. Q. Simultaneous Synthesis of $\mathrm{WO}_{3-x}$ Quantum Dots and Bundle-Like Nanowires Using a One-Pot Template-Free Solvothermal Strategy and Their Versatile Applications. Small 13, 1603689 (2017).

42. Zhang, P.; Mao, X. Y.; Mi, R.; Wang, L.; Yang, B. Mesoporous Silica Encapsulated Phosphotungstic Acid Catalysts for Alkylation Desulfurization of Gasoline. Catal. Lett. 151, 95-106 (2021).

43. Baibarac, M.; Baltog, I.; Frunza, S.; Magrez, A.; Schur, D.; Zaginaichenko, S. Y. Single-walled carbon nanotubes functionalized with polydiphenylamine as active materials for applications in the supercapacitors field. Dim. Relat. Mater. 32, 72-82 (2013).

44. Yu, H. J.; Chen, F.; Li, X. W.; Huang, H. W.; Zhang, Q. Y.; Su, S. Q.; Wang, K. Y.; Mao, E. Y.; Mei, B.; Mul, G.; Mai, T. Y.; Zhang, Y. Synergy of ferroelectric polarization and oxygen vacancy to promote $\mathrm{CO}_{2}$ photoreduction. Nat. Commun. 12, 4594 (2021).

45. Hu, J. D.; Chen, D. Y.; Mo, Z.; Li, N. J.; Xu, Q. F.; Li, H.; He, J. H.; Xu, H.; Lu, J. M. Z-Scheme 2D/2D Heterojunction of Black Phosphorus/Monolayer $\mathrm{Bi}_{2} \mathrm{WO}_{6}$ Nanosheets with Enhanced Photocatalytic Activities. Angew. Chem. Int. Ed. 58, 2073-2077 (2019).

46. Hanlon, D.; Backes, C.; Doherty, E.; Cucinotta, C. S.; Berner, N. C.; Boland, C.; Lee, K.; Harvey, A.; Lynch, P.; Gholamvand, Z.; Zhang, S. F.; Wang, K. P.; Moynihan, G.; Pokle, A.; Ramasse, Q. M.; McEvoy, N.; Blau, W. J.; Wang, J.; Abellan, G.; Hauke, F.; Hirsch, A.; Sanvito, S.; O’Regan, D D.; Duesberg, G. S.; Nicolosi, V.; Coleman, J. N. Liquid exfoliation of solvent-stabilized few-layer black phosphorus for applications beyond electronics. Nat. Commun. 6, 8563 (2015).

47. Zhang, N.; Li, X. Y.; Liu, Y. F.; Long, R.; Li, M. Q.; Chen, S. M.; Qi, Z. M.; Wang, C. M.; Song, L.; Jiang, J.; Xiong, Y. J. Defective Tungsten Oxide Hydrate Nanosheets for Boosting Aerobic Coupling of Amines: Synergistic Catalysis by Oxygen Vacancies and Brønsted Acid Sites. Small 13, 1701354 (2017). 
48. Liu, Z. Q.; Lu, Z. Y.; Bosman, M.; Li, N.; Frankcombe, T. J.; Jia, G. H.; Tricoli, A.; Liu, Y.; Du, Y. P.; Yin, Z. Y. Photoactivity and Stability Co-Enhancement: When Localized Plasmons Meet Oxygen Vacancies in MgO. Small 14, 1803233 (2018).

49. Liu, Y.; Zhang, Z.; Fang, Y.; Liu, B.; Huang, J.; Miao, F.; Bao, Y.; Dong, B. IR-Driven Strong PlasmonicCoupling on Ag Nanorices/ $\mathrm{W}_{18} \mathrm{O}_{49}$ Nanowires Heterostructures for Photo/Thermal Synergistic Enhancement of $\mathrm{H}_{2}$ Evolution from Ammonia Borane. Appl. Catal. B Environ. 252, 164-173 (2019).

50. Xu, F. Y; Meng, K.; Cheng, B.; Wang, S. Y.; Xu, J. S.; Yu, J. G. Unique S-scheme heterojunctions in selfassembled $\mathrm{TiO}_{2} / \mathrm{CsPbBr}_{3}$ hybrids for $\mathrm{CO}_{2}$ photoreduction. Nat. Commun. 11, 4613 (2020).

51. Liu, L. J.; Jiang, Y. Q.; Zhao, H. L.; Chen, J. T.; Cheng, J. L.; Yang, K. S.; Li, Y. Engineering Coexposed $\{001\}$ and $\{101\}$ Facets in Oxygen-Deficient $\mathrm{TiO}_{2}$ Nanocrystals for Enhanced $\mathrm{CO}_{2}$ Photoreduction under Visible Light. ACS Catal., 6, 1097-1108 (2016).

52. Wang, Y.; Arandiyan H.; Scott, J.; Aguey-Zinsou, K. F.; Amal, R. Single Atom and Nanoclustered Pt Catalysts for Selective $\mathrm{CO}_{2}$ Reduction. ACS Appl. Energy Mater., 1, 6781-6789 (2018).

53. Kresse, G.; Furthmüller, J. Efficient Iterative Schemes for ab Initio Total-Energy Calculations Using a Plane-Wave Basis Set. Phys. Rev. B 54, 11169 (1996).

54. Kresse, G.; Joubert, D. From Ultrasoft Pseudopotentials to the Projector Augmented-Wave Method. Phys. Rev. B 59, 1758 (1999).

55. Perdew, J. P.; Burke, K.; Ernzerhof, M. Generalized Gradient Approximation Made Simple. Phys. Rev. Lett. 77, 3865 (1996).

56. Perdew, J. P.; Chevary, J. A.; Vosko, S. H.; Jackson, K. A.; Pederson, M. R.; Singh, D. J.; Fiolhais, C. Atoms, Molecules, Solids, and Surfaces: Applications of the Generalized Gradient Approximation for Exchange and Correlation. Phys. Rev. B, 46, 6671 (1992).

57. Monkhorst, H. J.; Pack, J. D. Special Points for Brillouin-Zone Integrations. Phys. Rev. B 13, 5188 (1976).

58. Grimme, S. Semiempirical GGA-Type Density Functional Constructed with a Long-Range Dispersion Correction. J. Comput. Chem. 27, 1787 (2006).

\section{Figures}



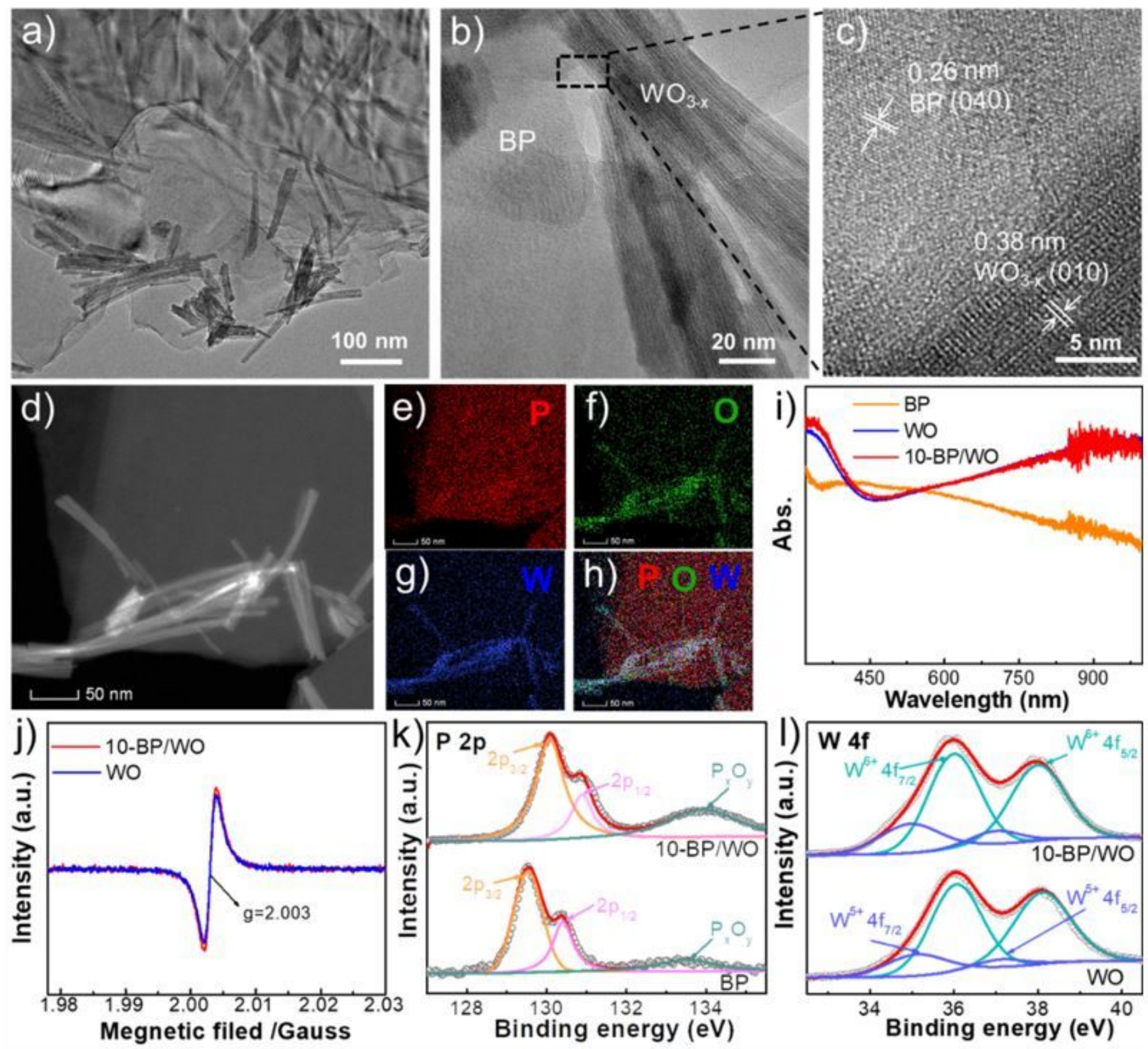

\section{Figure 1}

TEM (a, b), HR-TEM(c), HAADF-STEM (d) and elements mapping images (e-h) of 10-BP/WO heterostructures. $\mathrm{P}$ (red), $\mathrm{O}$ (green) and W (blue). UV-Vis-NIR diffuse reflectance spectra (DRS, i) and electron paramagnetic resonance (EPR) spectra (j) of WO, BP and 10-BP/WO heterostructures. X-ray photoelectron spectra (XPS) of element P 2p (k) and W $4 f(I)$ on sample BP, WO and 10-BP/WO, respectively. 
a)
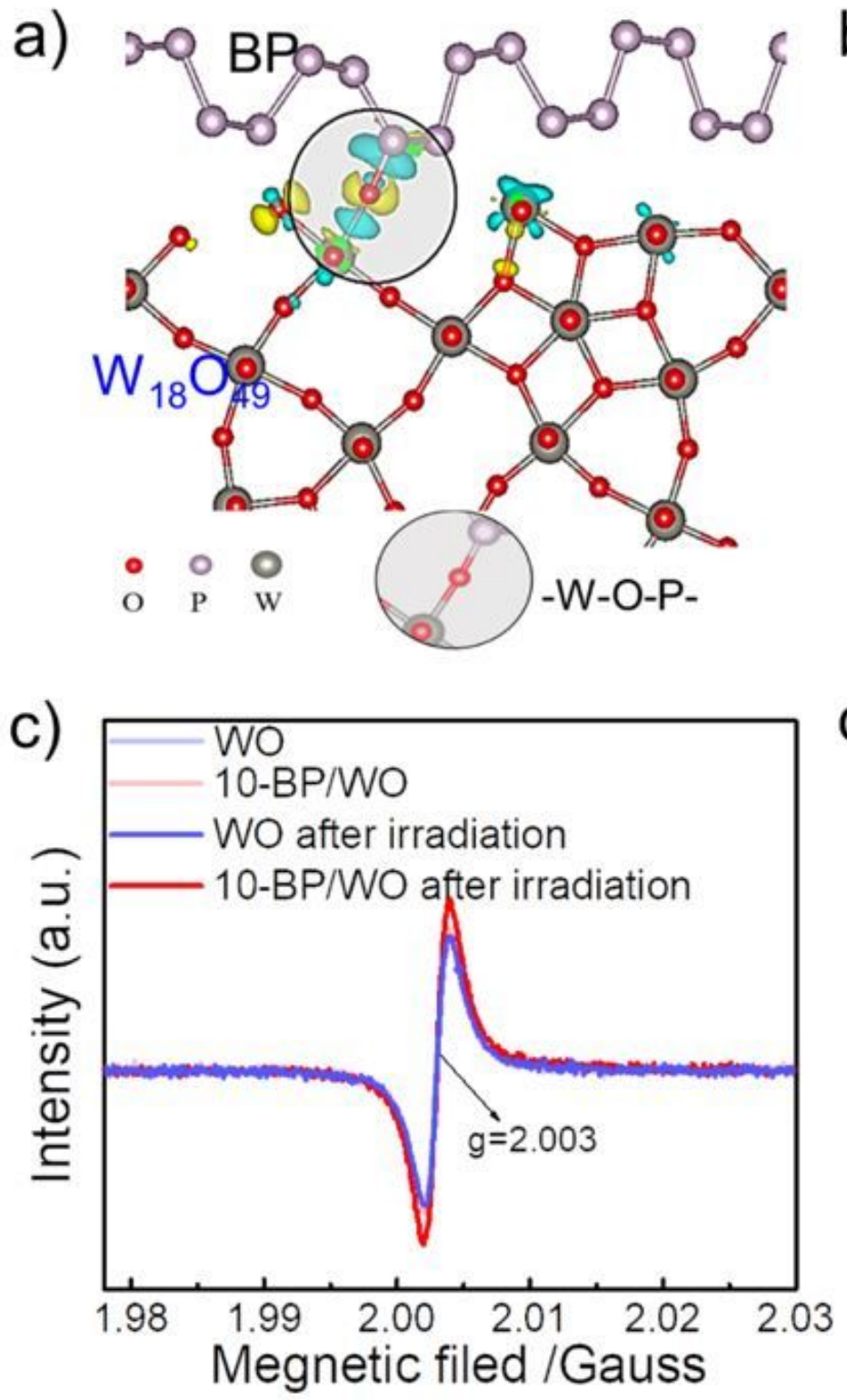

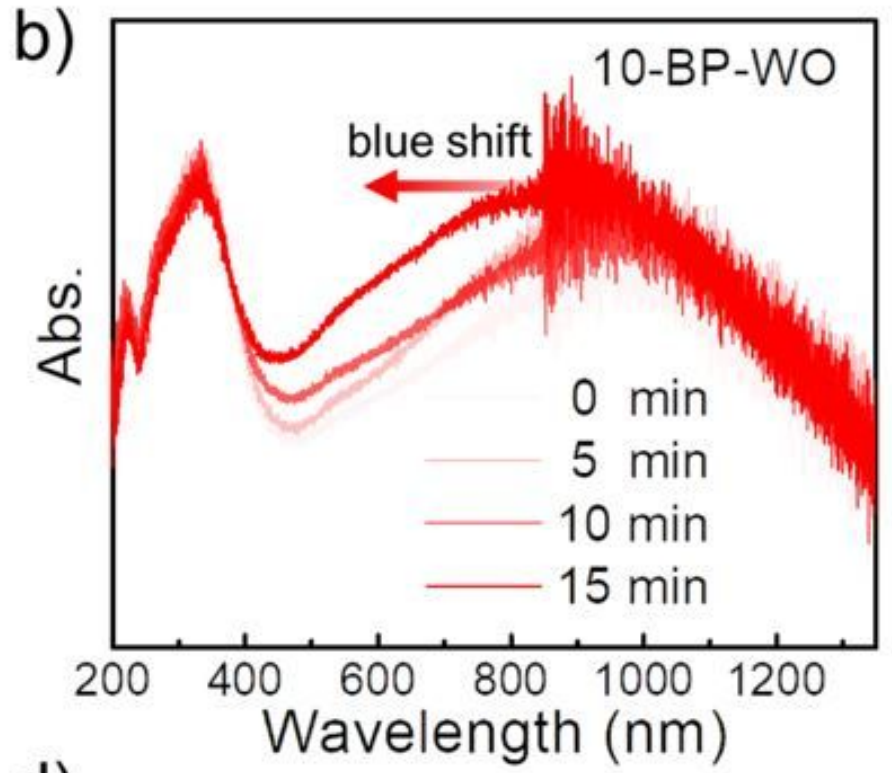

d)

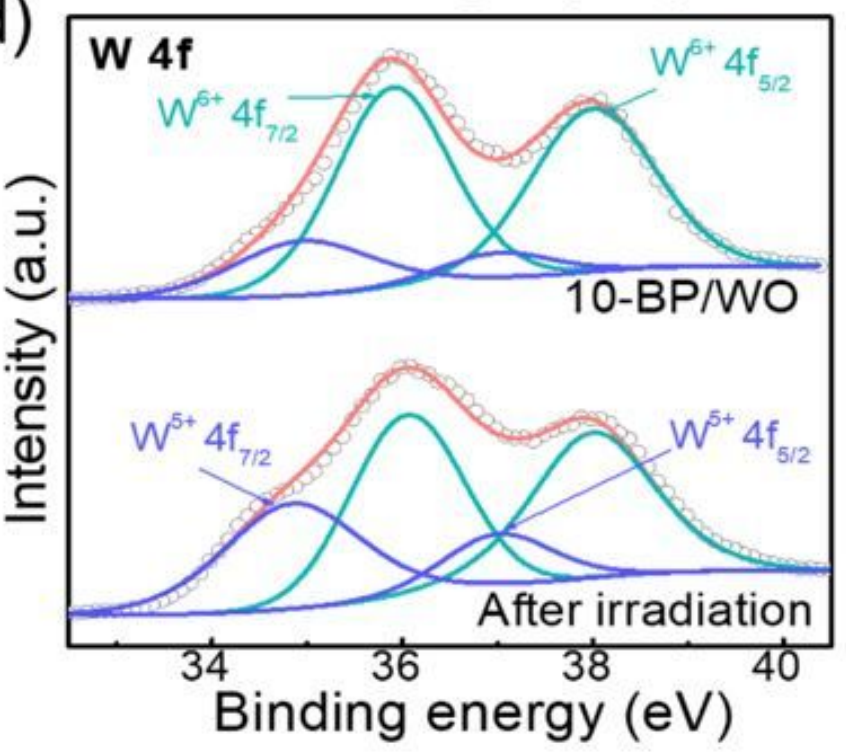

Figure 2

Top view of charge density difference (a) of black phosphorus/W18049(001) heterostructure. Blue and yellow isosurfaces represent charge accumulation and depletion in the space with respect to isolated W18049 (001) surface and black phosphorene. The isovalue is set as $0.53 \mathrm{e} / \mathrm{A} 3$. DRS (b) and EPR spectra (c) of 10-BP/WO heterostructure with different Vis-NIR light irradiation time. W $4 \mathrm{f} \mathrm{XPS} \mathrm{(d)} \mathrm{of} \mathrm{10-}$ BP/WO before and after Vis-NIR light irradiation. 

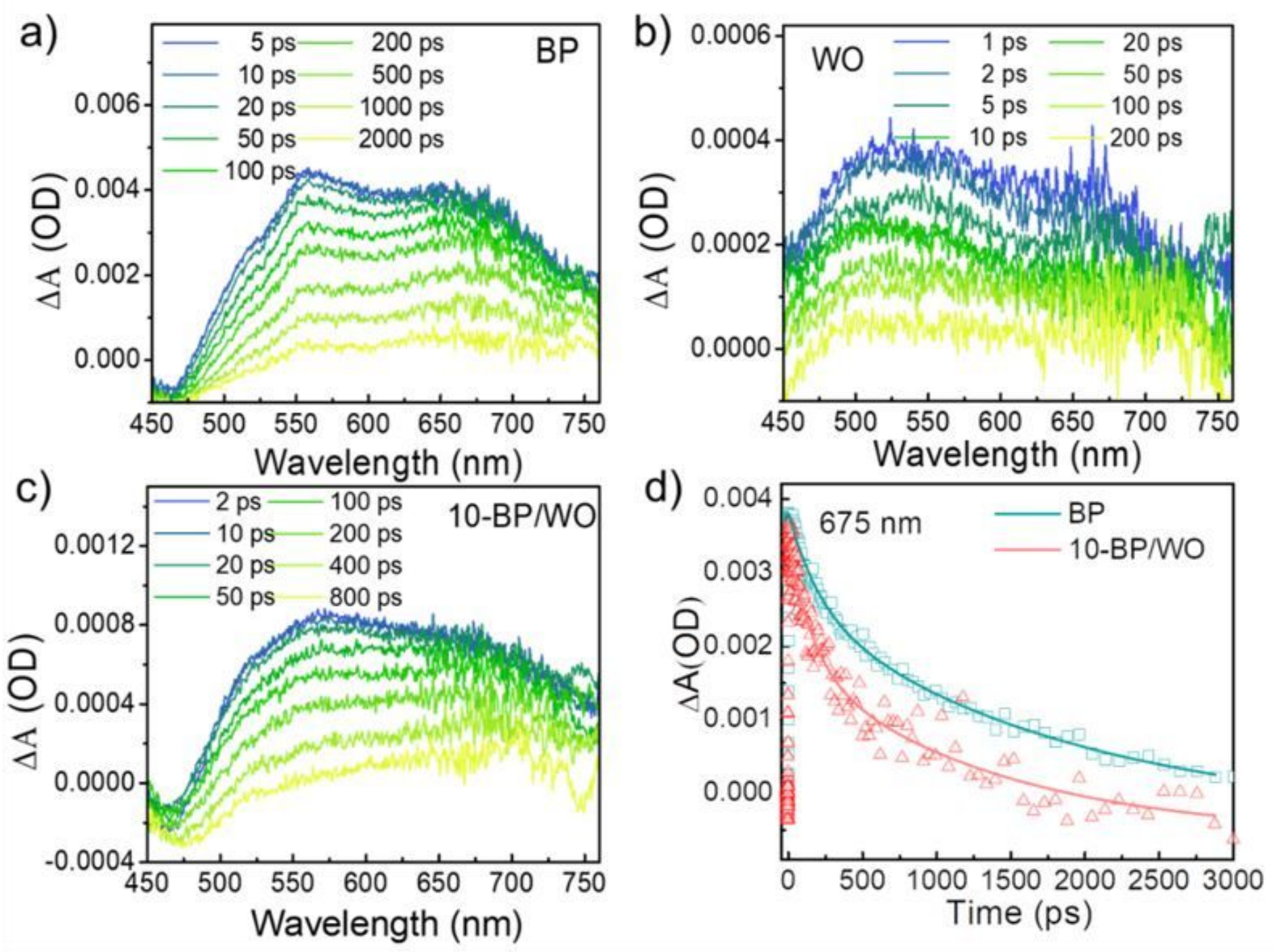

Figure 3

Ultrafast transient absorption spectra of BP (a), WO (b) and 10-BP/WO (c), respectively. The corresponding transient absorption kinetic curves (d) on BP and 10-BP/WO collected at $\lambda$ pump=675. 

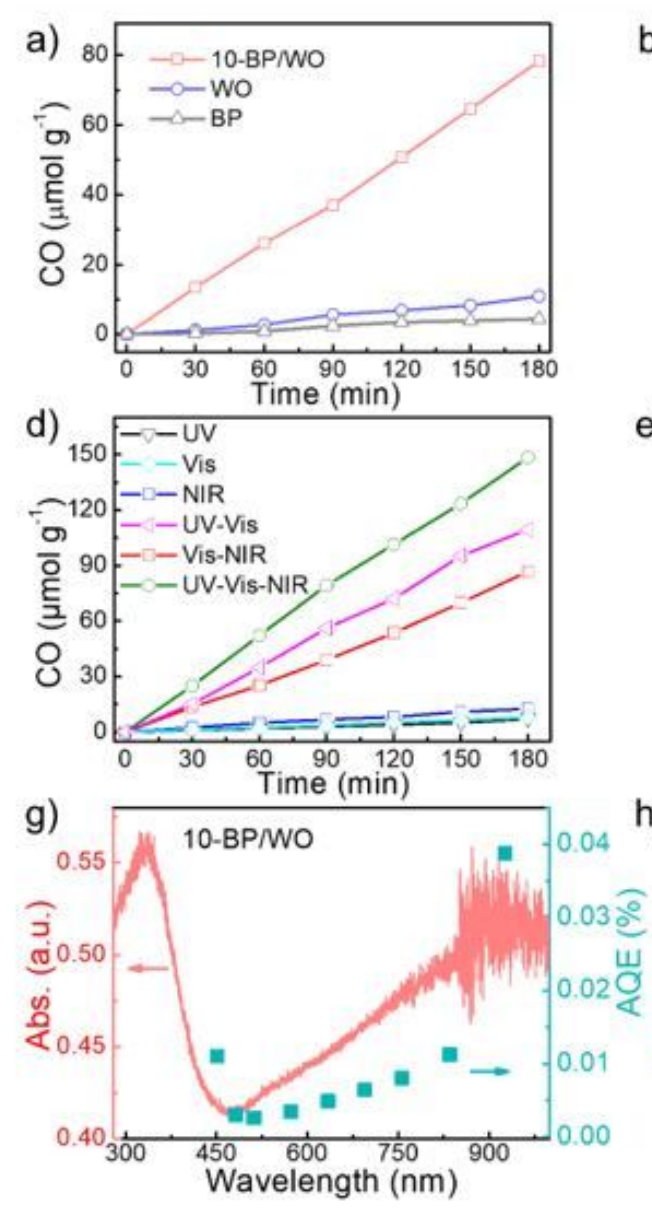

b)

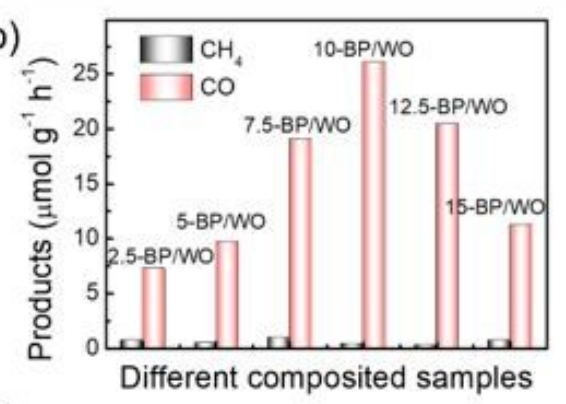

e)

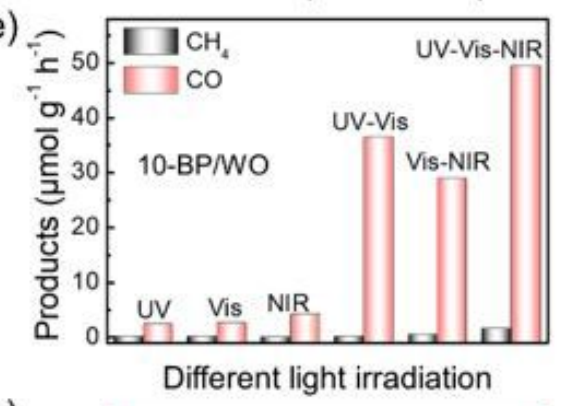

h)

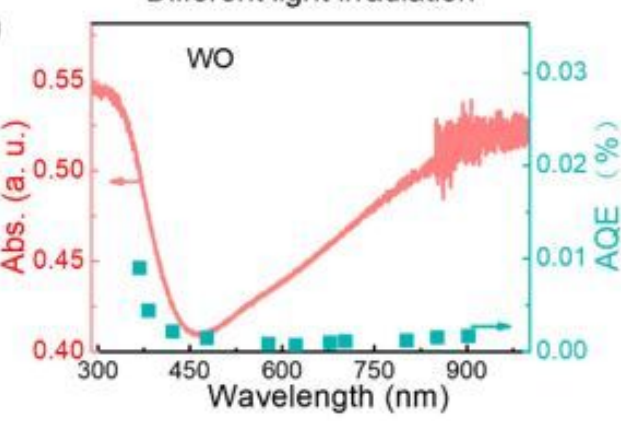

c)
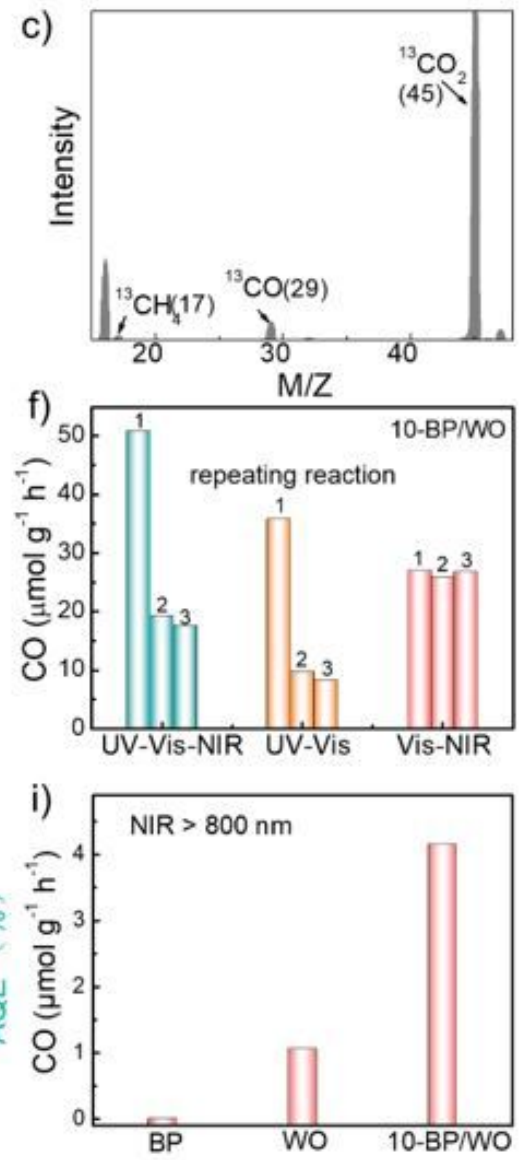

Figure 4

Photocatalytic CO generation (a) over BP, WO and 10-BP/WO heterostructures as catalysts during CO2-RR under Vis-NIR light irradiation. Products generation rates (b) over BP/WO heterostructures with different compositions. Mass spectrometry (MS) spectra (c) of products during CO2-RR by using 13C-isotope labeled $13 \mathrm{CO} 2$ as reactants. Photocatalytic $\mathrm{CO}$ generation (d) and products generation rates (e) over 10BP/WO as catalyst different light irradiation: UV (200-420 nm), Vis (420-780 nm), NIR (>780 nm), UV-Vis (200-780 nm), Vis-NIR (>420 nm) and UV-Vis-NIR. CO generation rates $(f)$ over 10-BP/WO during three times recycle CO2-RR under UV-Vis-NIR, UV-Vis and Vis-NIR light irradiation, respectively. Apparent quantum yield (AQE) of 10-BP/WO (g) and WO $(\mathrm{h})$ during photocatalytic CO2-RR. CO generation rates (a) over BP, WO and 10-BP/WO under NIR light (>800 nm) irradiation. Different irradiated light irradiation is supplied by the $300 \mathrm{~W}$ xenon lamp equipped with different cut-off filters. 

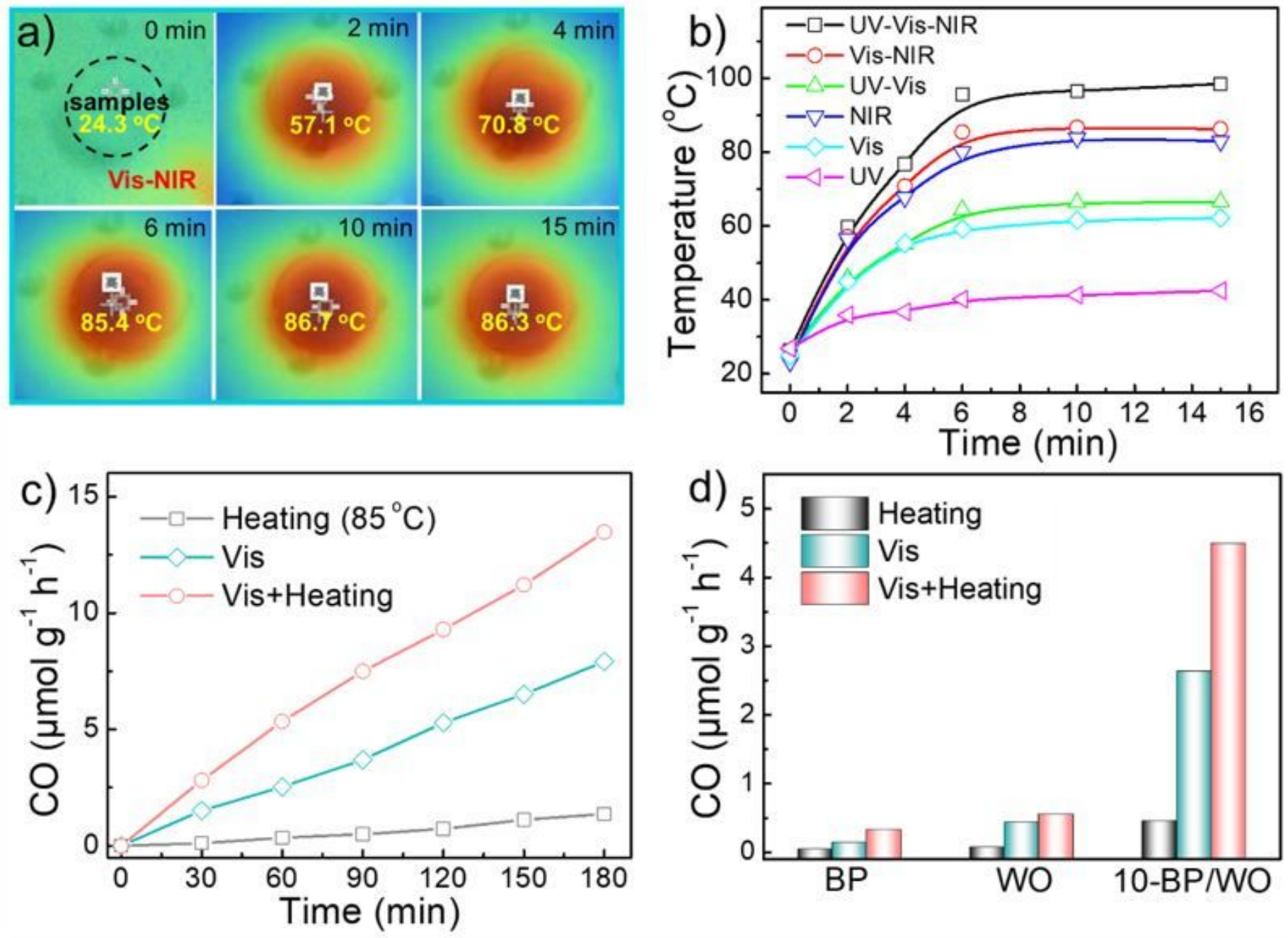

Figure 5

Thermal images (a) of 10-BP/WO heterostrucutres during photocatalysis under Vis-NIR-irradiation. The temperature changes (b) on 10-BP/WO heterostructures under different light irradiations: UV, Vis, NIR, UVVis, Vis-NIR and UV-Vis-NIR light, respectively. CO generation (c, d) over 10-BP/WO, WO and BP as catalysts under heating $(85 \mathrm{oC})$, Vis-irradiation $(420-780 \mathrm{~nm})$ and Vis-irradiation/heating, respectively. 

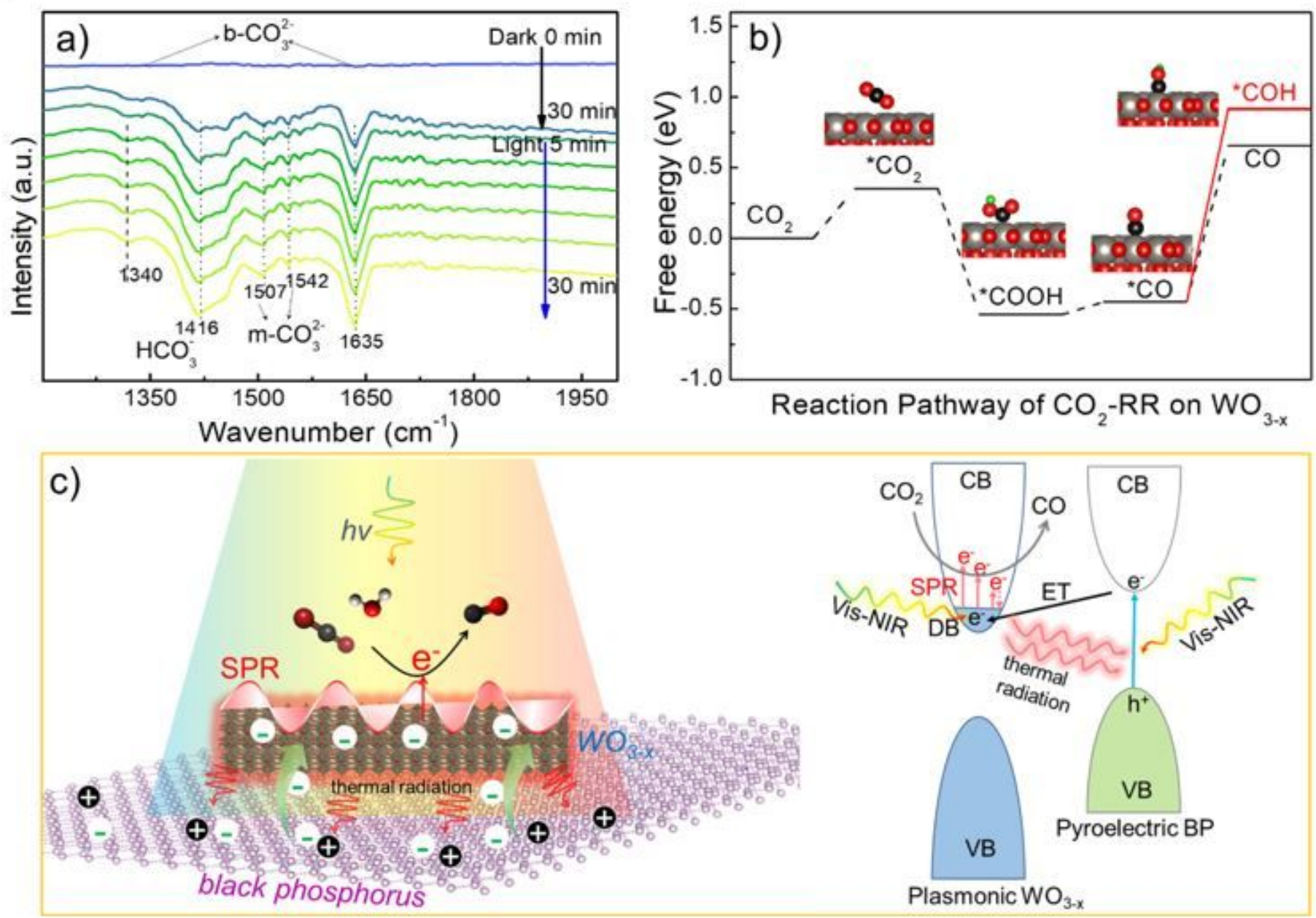

\section{Figure 6}

In situ FTIR spectra (a) of 10-BP/WO during photocatalytic CO2-RR. The possible reaction pathway (b) of CO2-RR on WO proposed based on the free energy computations by using DFT calculations. Schematic diagram (c) to illustrate the multi-synergetic processes (left) and possible photocatalytic mechanism (right) on plasmonic BP/WO heterostructures. ET (electron transfer), DB (defect band), VB (valence band), $\mathrm{CB}$ (conduction band).

\section{Supplementary Files}

This is a list of supplementary files associated with this preprint. Click to download.

- SupportingInformationNC.docx 